\title{
Research on the Architecture of Sky-Earth Collaborative Information Communication Network of GEI
}

\author{
Lan $\mathrm{Su}^{1}$, Peizhe Xin ${ }^{1}$, Yudong Wang ${ }^{1}$, Yugan Yang ${ }^{2}$, Chenchang Zhang ${ }^{2}$ and Geng Zhang ${ }^{3}$ \\ ${ }^{1}$ State Power Economic Research Instisute, Beijing 100052, China \\ ${ }^{2}$ Chongqing University, Chongjing 400040, China \\ ${ }^{3}$ Electric Power Research Institute, Beijing 100192, China
}

\begin{abstract}
Aiming at the significant demand of GEI in ultra high voltage(UHV), ubiquitous smart grid, the use of the regenerative clean energy, the paper presents a new sky-earth collaborative information communication network architecture which delivers the centralized and distributed power, wind energy, solar energy, ocean energy and other renewable energy to the global users. In order to ensure the wide service range, strong configuration ability, safe and reliable, green and low-carbon global energy allocation of GEI, it also the system studies topological structure, layered protocol and information and communication technology architecture to achieve the sky-earth collaborative information communication top-level architecture of GEI.
\end{abstract}

Keywords-GEI; sky-earth coordination; information communication; architecture

\section{The TARget And Business AnALysis Of GeI CONSTRUCION}

Energy and electricity are two important factors in the economic and society development in future. In the $21 \mathrm{st}$ century, continued increasing of global energy consumption and the exploited fossil energy result in many challenges in resource constraints, environmental pollution and climate change. In order to cope with this challenge, the global energy pattern needs deeply and thoroughly changed. Based on idea and practice, the Chairman of the state grid of China, Zhenya Liu has proposed the concept of "Global Energy Internet(GEI),"at the meeting of IEEE PES 2014.Global energy internet aims to promote the transformation of energy development, enable o get rid of resources, space and environmental constraints. There will help to achieve the clean energy, development, utilization, enable clean energy becoming the dominant energy, increase the proportion of clean energy consumption, promote the diversification of energy structure in China, even all over the world, and bring continued strong momentum for economic and social development.

EGI is a new energy network with developing and using large-scale energy such as pole, desert, marine, based on the UHV, providing reliable and real-time communication for large-scale, highly dynamic distributed energy .Specially in the complex natural environmentone as "pole-equator", the existing communication is difficult to meetting the demand. It needs to build a new information communication system architecture. At present, the electric power communication network is mainly based on optical fiber, which has formed a complete ground network. public/private wireless network, satellite communication has been applied in the power system. Since overcoming the geographical and time constraints, satellite has advantages of large covering area, operation stable and reliable communication quality and geographical barriers unconstraints. and so it is used in emergency communications. Now the $4 \mathrm{G} / 5 \mathrm{G}$ has be improved, and the cost of satellite is lower, improving network carrying capacity. The sky-earth integrated network gradually becomes preferred solution of GEI. In order to meet GEI business access, realize the wide range interconnect, real-time, efficient, safe and reliable work of the communication network, based on power trading platform, cloud platform and big data information technology, it is necessary to construct a communication architecture to effectively adapt to four and to support wide area energy interconnect. The GEI communication architecture is shown in Figure1.

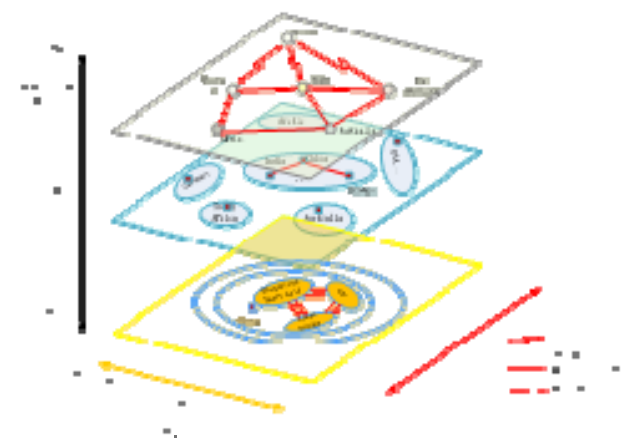

FIGURE I. GEI COMMUNICATION SERVICE PLATFORM THREE-DIMENSIONAL MAP

\section{GLOBAL ENERGY INTERNET INFORMATION COMMUNICATION NETWORK TOPOLOGY AND SYSTEM COMPOSITION}

Facing complex business system of GEI, information communication network must effectively carry various forms of business, realize deep coupling of the information flow and energy flow, and provide reliable support for access, transmission and management[4]. Based on the analysis of national and global energy power supply demand strategy, and 
major demand of power flow, "pole-equator" and the large-scale clean energy base development, the paper proposes a heterogeneous communication network topology under GEI, as shown in Figure 2:

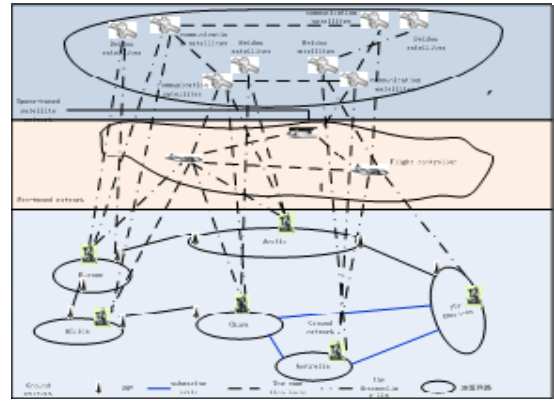

FIGURE II. TOPOLIGY OF GLOBAL INTERNET WORD ENERGY COOPERATIVE COMMUNICATION NETWORK

Ground-space-aeronautics integrated heterogeneous communication network of GEI is composed of the ground network, the near space network and space-based network, which is an open, $\mathrm{k}$ architecture. The ground network is mainly composed by long distance fiber (along with UHV laying), long distance wireless $(4 \mathrm{G} / 5 \mathrm{G})$, short distance wireless, and satellite ground station (routers, gateways and encryption machine, etc.)For example, Energy information can be transmitted by submarine cable and the optical fiber transmission, or directly connected to the satellite ground station in the arctic regions. The near space network is to provide seamless monitoring and IC services, providing alternative data protection for electric power data security, assisting to complete satellite communication. In order to achieve connection of global energy information, GEI construction need to depend on iridium satellites system and global satellites system, which have realized the global coverage.

Ground-space-aeronautics integrated communication network of GEI world cooperative communication network consists of multiple satellites in different orbits, using intelligent high-speed on-board processing and switching and routing technology, for microwave, optical and infrared multi spectral information, according to the information resources of the maximum effective comprehensive utilization principle, information access to accurate, fast processing and high efficient transmission of high-speed broadband integrated high capacity information network.

\section{Analysis On The Layered Protocol Of The GROUND-SPACE-AERONAUTICS INTEGRATED COMMUNICATION NETWORK}

In order to build a real time and efficient ground-space-aeronautics integrated communication network, the network protocol model is adopted in Figure 3, which is based on the design principle of considering general purpose and special usage. The network protocol is divided into four levels, which are application layer, network layer, link layer and physical layer. Each level includes common protocols and task oriented special protocol, supporting the communication needs of normal application and key task[5].

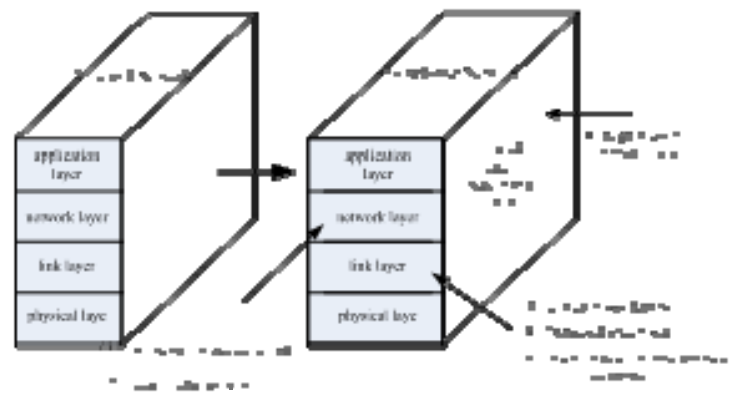

FIGURE III. THE PROTOCOL MODEL OF GLOBAL ENERGY COOPERATIVE INTERNET WORD

information and communication network

The network protocol model of ground-space-aeronautics integrated communication network is similar to common network, and the network protocol is also divided into four layers, namely, physical layer, link layer, network layer and application layer. Physical layer describes the physical channel parameters of communication, including the frequency band, bandwidth, antenna polarization, modulation, encoding and the basic communication parameters. Link layer is mainly to solve the access between vehicle nodes with the space-based network, and link control for data transmission of special power network, including link control for aircraft node and probe information transmission. The network layer provides relay and routing functions, mainly uses the following two routing protocols:1) the static routing protocol of the permanent link, 2) quasi-static routing protocol. Application layer provides cooperative detection application service mechanism and key task service protocol.

\section{THE INFORMATION COMMUNICATION TECHNOLOGY SYSTEM OF SUPPORTING GEI}

Complex business of relay protection, fault isolation and power flexible selling increases multidimensional integration requirements. Multidimensional IC platform of technologies integration, real-time transmission, management and Scheduling has increased the requirements completeness and systematization. Building a complete, top-level communication network technology system to meet business needs is an important support to promote the development. The system architecture is shown in figure 4 :

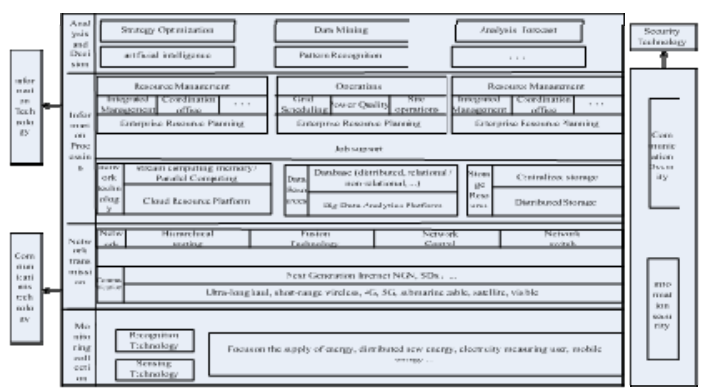

FIGURE IV. GLBLAL ENERGY INTERNET ICT ARCHITECTURE DIAGRAM

The GEI information communication technology 
architecture mainly includes three parts: communication, information and security, the follows are some detail introductions[2].

Station communications generally refers to use wireless sensor technology transmission the infomantion of monitoring and control to the control room. Especially in unattended station, this network is not only high autonom, but also high bandwidth and anti-electromagnetic interference capability, providing a good secure environment for transmission. External communication in use ultra-long optical fiber with UHV or submarine cable paved. It uses supplementary of private/public wirelessnetwork without fiber.The place in acrossing continents or oceans, it chooses satellite transmission in cooperation with ground communication. Because satellite transmission has big delay, limit bandwidth priority, it selects the correct constellation design method, frequency range and security method. Network control is a means to make a variety of heterogeneous communication co-operation, generally uses the interoperability and integration of space technology, hierarchical routing protocol, network optimization control technology and network switching technology. Multi network fusion technology generally is data multiplexing, multi-point collaboration, joint processing technology. Switching technology generally is horizontal handover, vertical handover, soft handover, hard handover. 。 Hierarchical routing protocols is adapt to network topology changing, with a strong anti-destruction and quickly recover capability.

(2)Information includes: information processing and analysis-ecision technology. Information processing is to support the effective, reliable transmission and fast deployment of underlying data. As the foundation of the cloud computing platform, flow, memory and parallel calculation provide computing resources; SDN applications in globalenergy internetworkt, observe the health of the whole network, and make timely decisions and instructions issued under the early warning; Data resource pool consists of database technology big data platform, geographic information, high-performance implementation of energy data migration and storage, maximizing network performance[1].

(3)Safety class includes: communications and information security. A single point attacktion and mobile attack need to take protective measures, such as quantum security technology, improve the bottom (physical layer, data link layer)transmission protocol algorithm and others to disintegrate pseudo base stations and terminals. Trusted protective measures include: Authentication is used to determine the identity of legality; Encryption/decryption is to information algorithm protection in transport and storage; Access control technology is to network resources from unauthorized use and access; Security audit is to analyze security attacks trajectory. detection monitoring technology, information network traffic or application content for multi-level detection and appropriate regulation and control, to avoid the abuse of network traffic, garbage information and dissemination of harmful information, such as data filtering technology.

\section{CONCLUSIONS}

In order to achieve the connection in continent, intercontinental and global energy, the architecture of sky-earth collaborative information communication network is the onlychoice.It defines new business and functional requirements, key technologies, topology model and architecture, to providing for layout, operation mode and recommendations., to layingba foundation for standard. It designes a more clear roadmap for top-level for further Studies. and changes strategic into reality.

\section{REFERENCES}

[1] C.M. Torres-Ortolaza, A.A. Irizarry-Riviera, Reliability of intelligent power routers, in: 2004 International Conference on Probabilistic Methods Applied to PowerSystems,2004,pp.838-843.

[2] Hongtao Liu, Zheng $\mathrm{Xu}$, Ying Huang, Study of protection strategy for VSC based HVDC system, in: Transmission and Distribution Conference and Exposition, IEEE $\mathrm{P}$

[3] Cao Junwei, Meng Kun, Wang Jiye, et al. An energy internet and energy routers $[J]$. Scientia Sinica Informationis,2014,44(6) : 714-727(in Chinese).

[4] Wang Jiye, Meng Kun, Cao Junwei, et al. Research on information technology for energy internet: a survey $[\mathrm{J}]$. ournal of Computer Research and Development,2015,52(3): 1-18(in Chinese).

[5] Lee E. Computing foundations and practice for cyber-physical systems: a prelim-inary report[R].Berkeley, America: University of California, 2007.

[6] Dong Zhaoyang, Zhao Junhua, Wen Fushuan, et al. From smart grid to energy internet : the basicconcept and research framework[J] . Automation of Electric Power Systems, 2014, 38(15): 1-11(in Chinese).

[7] Wang X W, Dang Q, Guo J L, et al. RFID application of smart grid for asset management[J] .International Journal of Antennas and Propagation, 2013, 2013.

[8] Zhang Dongxia, Miao Xin, Liu Liping, et al. Research on development strategy for smart grid big data[J]. Proceedings of the CSEE, 2015, 35(1): 2-12(in Chinese).

[9] Xu Kehang. Application of wireless communication technology in power system[J]. Telecom World, 2013(21): 122-123(in Chinese).

[10] Qi Xiaoxiao. Application of data mining on transient stability assessment in power system[D].Xian: Xi'an University of Technology, 2005(in Chinese). 